\title{
On Uniform Exponential Stability and Exact Admissibility of Discrete Semigroups
}

\author{
Aftab Khan, ${ }^{1,2}$ Gul Rahmat, ${ }^{2}$ and Akbar Zada ${ }^{3}$ \\ ${ }^{1}$ Shaheed Benazir Bhutto University Sheringal, Dir Upper 18000, Pakistan \\ ${ }^{2}$ Government College University, Abdus Salam School of Mathematical Sciences (ASSMS), Lahore 54600, Pakistan \\ ${ }^{3}$ Department of Mathematics, University of Peshawar, Peshawar 25000, Pakistan
}

Correspondence should be addressed to Akbar Zada; zadababo@yahoo.com

Received 22 April 2013; Revised 19 June 2013; Accepted 20 June 2013

Academic Editor: Sotiris Ntouyas

Copyright (C) 2013 Aftab Khan et al. This is an open access article distributed under the Creative Commons Attribution License, which permits unrestricted use, distribution, and reproduction in any medium, provided the original work is properly cited.

We prove that a discrete semigroup $\mathbb{T}=\left\{T(n): n \in \mathbb{Z}_{+}\right\}$of bounded linear operators acting on a complex Banach space $X$ is uniformly exponentially stable if and only if, for each $x \in A P_{0}\left(\mathbb{Z}_{+}, X\right)$, the sequence $n \mapsto \sum_{k=0}^{n} T(n-k) x(k): \mathbb{Z}_{+} \rightarrow X$ belongs to $A P_{0}\left(\mathbb{Z}_{+}, X\right)$. Similar results for periodic discrete evolution families are also stated.

\section{Introduction}

The solutions of the autonomous discrete systems $x_{n+1}=A x_{n}$ or $y_{n+1}=A y_{n}+h_{n}$ lead to the idea of discrete semigroups. There are a lot of spectral criteria which characterize different types of stability (or other types of asymptotic behavior) of the solutions of above systems. For further results on asymptotic behavior of semigroups, we refer to [1].

New difficulties appear in the study of the nonautonomous systems, especially because the part of the solution generated by the forced term $\left(h_{n}\right)$, that is, $\sum_{k=\gamma}^{n} U(n, k) h_{k}$, is not a convolution in the classical sense. These difficulties may be passed by using the so-called evolution semigroups.

The evolution semigroups were exhaustively studied in [2]. Having in mind the well-known results stated in the continuous case, see for example [2, 3], we can say that this method is a very efficient one. See also $[4,5]$ for recent developments concerning the semigroups of evolution acting on almost periodic function spaces.

Recently, the discrete version of [6] was obtained in [7].

In this note, we study the asymptotic behavior of the discrete semigroups in terms of exact admissibility of the space of almost periodic sequences.

In this regard, we develop the theory of discrete evolution semigroups on a special space of bounded sequences. Results of this type in the continuous case may be found in [8] and the references therein. However, by contrast with the continuous case, we did not find in the existent literature papers written in the spirit of the present one referring to the discrete evolution semigroups. These results could be new and useful for people whose area of research is restricted to difference equations.

\section{Definitions and Preliminary Results}

Let $X$ be a complex Banach space and $\mathscr{B}(X)$ the Banach algebra of all linear and bounded operators acting on $X$. The norms in $X$ and in $\mathscr{B}(X)$ will be denoted by $\|\cdot\|$. Let $\mathbb{Z}_{+}$ be the set of all nonnegative integer numbers. A sequence $x: \mathbb{Z}_{+} \rightarrow X$ is said to be almost periodic if for any $\epsilon>0$ there exists an integer $l_{\epsilon}>0$ such that any discrete interval of length $l_{\epsilon}$ contains an integer $\tau$, such that

$$
\left\|x_{n+\tau}-x_{n}\right\| \leq \epsilon, \quad \forall n \in \mathbb{Z}_{+} .
$$

The integer number $\tau$ is called $\epsilon$-translation number of $\left(x_{n}\right)$. The set of all almost periodic sequences will be denoted by $A P\left(\mathbb{Z}_{+}, X\right)$. For further details about almost periodic functions, we refer to the books $[9,10]$. The set $l^{\infty}\left(\mathbb{Z}_{+}, X\right)$ of all bounded sequences becomes a Banach space when it is endowed with the "sup" norm denoted by $\|\cdot\|_{\infty}$. Clearly, $A P\left(\mathbb{Z}_{+}, X\right)$ is a subset of $l^{\infty}\left(\mathbb{Z}_{+}, X\right)$. Let $P_{q}^{0}\left(\mathbb{Z}_{+}, X\right)$ be the space of all $q$-periodic ( $q \geq 2$ is an integer number) sequences 
$x$ with $x(0)=0$. Denote by $\mathscr{A}_{0}\left(\mathbb{Z}_{+}, X\right)$ the set of all sequences $\{x(n)\}_{n \geq 0}$ for which there exists $n_{x} \in \mathbb{Z}_{+}$with $n_{x}>0$ and $y_{x} \in P_{q}^{0}\left(\mathbb{Z}_{+}, X\right)$ such that

$$
x(n)= \begin{cases}0, & \forall 0 \leq n<n_{x} \\ y_{x}(n), & \text { if } n \geq n_{x} .\end{cases}
$$

Let $A P_{0}\left(\mathbb{Z}_{+}, X\right):=\overline{\operatorname{span}}\left\{\mathscr{A}_{0}\left(\mathbb{Z}_{+}, X\right)\right\}$. Here the closeness is considered in the space $l^{\infty}\left(\mathbb{Z}_{+}, X\right)$.

For a bounded linear operator $L$, acting on $X$, we denote by $\sigma(L)$ the spectrum of $L$ and by $\rho(L)$ its resolvent set. Recall that a subset $\mathbb{Z}=\{T(n)\}_{n \in \mathbb{Z}_{+}}$of $\mathscr{B}(X)$ is called discrete semigroup if it satisfies the following conditions:

(i) $T(0)=I$, where $I$ is the identity operator on $X$.

(ii) $T(n+m)=T(n) T(m)$, for all $n, m \in \mathbb{Z}_{+}$.

A discrete semigroup $\mathbb{\mathbb { T }}$ is said to be uniformly exponentially stable if there exist $N, v>0$ such that

$$
\|T(n)\| \leq N e^{-v n} \quad \forall n \in \mathbb{Z}_{+} .
$$

The spectral radius of $T(1)$ denoted by $r(T(1))$ is defined as

$$
r(T(1)):=\sup \{|\lambda|: \lambda \in \sigma(T(1))\} .
$$

It is well known that, see for example [11, page 42],

$$
r(T(1))=\lim _{n \rightarrow \infty}\left\|(T(1))^{n}\right\|^{1 / n} .
$$

As a consequence of (5), a discrete semigroup $\{T(n)\}_{n \in \mathbb{Z}_{+}}$is uniformly exponentially stable if and only if $r(T(1))<1$.

Having in mind the continuous case, the "infinitesimal generator" of the discrete semigroup denoted by $G$ is defined by $G:=T(1)-I$. For discrete semigroups, the Taylor formula of order one is

$$
T(n) x-x=\sum_{k=0}^{n-1} T(k) G x, \quad \forall n \in \mathbb{Z}_{+}, n \geq 1, \quad \forall x \in X .
$$

A discrete semigroup $\mathbb{T}$ is said to be $A P_{0}\left(\mathbb{Z}_{+}, X\right)$ exact admissible, if for every $x \in A P_{0}\left(\mathbb{Z}_{+}, X\right)$ the sequence $\left(\sum_{k=0}^{n} T(n-k) h(k)\right)_{n \in \mathbb{Z}_{+}}$belongs with $A P_{0}\left(\mathbb{Z}_{+}, X\right)$.

The evolution semigroup $\mathbb{S}=\left\{S(n), n \in \mathbb{Z}_{+}\right\}$associated with $\mathbb{T}$ on $A P_{0}\left(\mathbb{Z}_{+}, X\right)$ is defined by

$$
(S(r) x)(n)= \begin{cases}T(r) x(n-r), & \forall n \geq r, \\ 0, & 0 \leq n \leq r .\end{cases}
$$

\section{Results}

The following lemma shows that the associated evolution semigroup $\{S(n)\}_{n \in \mathbb{Z}_{+}}$acts on $A P_{0}\left(\mathbb{Z}_{+}, X\right)$.

Lemma 1. Let $x \in A P_{0}\left(\mathbb{Z}_{+}, X\right)$ and $\mathbb{T}=\{T(j)\}_{j \in \mathbb{Z}_{+}}$be a discrete semigroup of bounded linear operators on $X$. The sequence $S(r) x$, given by

$$
(S(r) x)(n)= \begin{cases}T(r) x(n-r), & \forall n \geq r \\ 0, & 0 \leq n \leq r,\end{cases}
$$

belongs to $A P_{0}\left(\mathbb{Z}_{+}, X\right)$.
Proof. First we show that $S(r) x \in \mathscr{A}_{0}\left(\mathbb{Z}_{+}, X\right)$ for any $x \in$ $\mathscr{A}_{0}\left(\mathbb{Z}_{+}, X\right)$. Since $x \in \mathscr{A}_{0}\left(\mathbb{Z}_{+}, X\right)$ there exist $n_{x} \in \mathbb{Z}_{+}$with $n_{x}>0$, and $\left(y_{x}(n)\right) \in P_{q}^{0}\left(\mathbb{Z}_{+}, X\right)$, such that

$$
x(n)= \begin{cases}0, & \text { if } 0 \leq n<n_{x} \\ y_{x}(n), & \text { if } n \geq n_{x}\end{cases}
$$

Let $n_{S(r) x}:=r+n_{x}$ and set $y_{S(r) x}(\cdot)=T(r) y_{x}(\cdot-r)$. Clearly $y_{S(r) x}$ is $q$-periodic sequence. It remains to show that

$$
(S(r) x)(n)= \begin{cases}0, & \text { if } 0 \leq n<n_{S(r) x} \\ y_{S(r) x}(n), & \text { if } n \geq n_{S(r) x}\end{cases}
$$

If $n \leq n_{S(r) x}=r+n_{x}$, then $n-r<n_{x}$ and $x(n-r)=0$, so

$$
(S(r) x)(n)=T(r) x(n-r)=0 .
$$

If $n \geq n_{S(r) x}=r+n_{x}$, then $n-r \geq n_{x}$ and $x(n-r)=y_{x}(n-r)$; hence

$$
\begin{aligned}
(S(r) x)(n) & =T(r) x(n-r) \\
& =T(r) y_{x}(n-r) \\
& =y_{S(r) x}(n) .
\end{aligned}
$$

Thus $S(r) x \in \mathscr{A}_{0}\left(\mathbb{Z}_{+}, X\right)$. Now, from linearity it follows that $S(r) z$ belongs to $\operatorname{span}\left\{\mathscr{A}_{0}\left(\mathbb{Z}_{+}, X\right)\right\}$ whenever $z \in$ $\operatorname{span}\left\{\mathscr{A}_{0}\left(\mathbb{Z}_{+}, X\right)\right\}$. Let now $\epsilon>0, x \in A P_{0}\left(\mathbb{Z}_{+}, X\right)$, and let $z \in \operatorname{span}\left\{\mathscr{A}_{0}\left(\mathbb{Z}_{+}, X\right)\right\}$, such that $\|x-z\|_{l^{\infty}\left(\mathbb{Z}_{+}, X\right)}<\epsilon$. Clearly $S(r) z$ belongs to $\operatorname{span}\left\{\mathscr{A}_{0}\left(\mathbb{Z}_{+}, X\right)\right\}$, and

$$
\begin{aligned}
\|S(r) z-S(r) x\|_{l^{\infty}\left(\mathbb{Z}_{+}, X\right)} & =\sup _{n \geq r}\|T(r)[z(n-r)-x(n-r)]\| \\
& \leq M e^{v r} \sup _{n \geq r}\|z(n-r)-x(n-r)\| \\
& \leq M e^{v r} \epsilon,
\end{aligned}
$$

that is, $S(r) x$ is in $A P_{0}\left(\mathbb{Z}_{+}, X\right)$. This completes the proof.

Lemma 2. Let $\mathbb{T}=\{T(n)\}_{n \in \mathbb{Z}_{+}}$be a discrete semigroup of bounded linear operators on $X$, and let $\mathbb{S}=\left\{S(n), n \in \mathbb{Z}_{+}\right\}$ be the evolution semigroup associated with $\mathbb{1}$ on $A P_{0}\left(\mathbb{Z}_{+}, X\right)$, having $G_{\mathbb{S}}$ as generator. Let $x, z \in A P_{0}\left(\mathbb{Z}_{+}, X\right)$. The following two statements are equivalent:

(i) $G_{\mathbb{S}} x=-z$,

(ii) $x(n)=\sum_{k=0}^{n} T(n-k) z(k)$, for all $n \in \mathbb{Z}_{+}$.

Proof. (i) $\Rightarrow$ (ii): Using the Taylor formula (6), one has

$$
S(n) x-x=\sum_{m=0}^{n-1} S(m) G_{\mathbb{S}} x=-\sum_{m=0}^{n-1} S(m) z .
$$


Then, for every $n \in \mathbb{Z}_{+}$, one has

$$
\begin{aligned}
x(n) & =(S(n) x)(n)+\sum_{m=0}^{n-1}(S(m) z)(n) \\
& =T(n) x(0)+\sum_{m=0}^{n-1} T(m) z(n-m) \\
& =\sum_{k=0}^{n} T(n-k) z(k) .
\end{aligned}
$$

(ii) $\Rightarrow$ (i): For each $n \in \mathbb{Z}_{+}$, one has

$$
\begin{aligned}
\left(G_{\mathbb{S}} x\right)(n) & =(S(1)-I) x(n) \\
& =T(1) x(n-1)-x(n) \\
& =T(1) \sum_{k=0}^{n-1} T(n-1-k) z(k)-x(n) \\
& =\sum_{k=0}^{n-1} T(n-k) z(k)-\sum_{k=0}^{n} T(n-k) z(k) \\
& =-z(n) .
\end{aligned}
$$

This completes the proof.

See also [12], for a variant of this lemma in other space.

The next result is the main ingredient in the proof of Theorem 5 that follows.

Theorem 3 (see [7]). Let $\mathbb{T}=\left\{T(n): n \in \mathbb{Z}_{+}\right\}$be a discrete semigroup on $X$, and let $\mu$ be a real number. If

$$
\sup _{n \geq 0}\left\|\sum_{k=0}^{n} e^{i \mu k} T(n-k) f(k)\right\|<\infty,
$$

for every $f \in P_{0}^{q}\left(\mathbb{Z}_{+}, X\right)$, then $T(1)$ is power bounded (i.e., $\left.\sup _{n \in \mathbb{Z}_{+}}\left\|A^{n}\right\|<\infty\right)$ and $e^{i \mu} \in \rho(T(1))$.

As a corollary of this theorem, we state the following.

Corollary 4 (see [7]). Let $\mathbb{T}=\left\{T(n): n \in \mathbb{Z}_{+}\right\}$be a discrete semigroup on $X$. If the condition (17) holds for every $\mu \in \mathbb{R}$ and every $f$ in $P_{0}^{q}\left(\mathbb{Z}_{+}, X\right)$, then the semigroup $\mathbb{T}$ is uniformly exponentially stable.

The result of this paper reads as follows.

Theorem 5. Let $\mathbb{T}=\{T(n)\}_{n \in \mathbb{Z}_{+}}$be a discrete semigroup on $X$. The following four statements are equivalent:

(i) $\mathbb{T}$ is uniformly exponentially stable.

(ii) The evolution semigroup $\mathbb{S}$ associated with $\mathbb{T}$ on $A P_{0}\left(\mathbb{Z}_{+}, X\right)$ is uniformly exponentially stable.

(iii) The semigroup $\mathbb{T}$ is $A P_{0}\left(\mathbb{Z}_{+}, X\right)$ exact admissible.

(iv) $\sup _{n \in \mathbb{Z}_{+}}\left\|\sum_{k=0}^{n} T(n-k) z(k)\right\|=M_{z}<\infty$, for all $z \in$ $A P_{0}\left(\mathbb{Z}_{+}, X\right)$.
Proof. (i) $\Rightarrow$ (ii): Let $\mathbb{T}$ be uniformly exponentially stable, and let $N$ and $\nu$ be positive constants such that

$$
\|T(n)\| \leq N e^{-\nu n} \quad \forall n \in \mathbb{Z}_{+} .
$$

Then for every $f$ in $A P_{0}\left(\mathbb{Z}_{+}, X\right)$, one has

$$
\|S(j) f\|_{\infty}=\sup _{n \geq j}\|T(j) f(n-j)\| \leq N e^{-v j}\|f\|_{\infty} .
$$

(ii) $\Rightarrow$ (iii): Since $\mathbb{S}$ is uniformly exponentially stable, $1 \in \rho(S(1))$, that is, $S(1)-I$ is invertible. Then for each $z$ in $A P_{0}\left(\mathbb{Z}_{+}, X\right)$, there exists $u \in A P_{0}\left(\mathbb{Z}_{+}, X\right)$ such that $(S(1)-$ I) $u=-z$.

On the other hand, by Lemma $2, u(n)=\sum_{k=0}^{n} T(k) z(n-k)$, for every $n \in \mathbb{Z}_{+}$; hence $\mathbb{T}$ is $A P_{0}\left(\mathbb{Z}_{+}, X\right)$ exact admissible.

(iii) $\Rightarrow$ (iv) It is obvious.

(iv) $\Rightarrow$ (i) Obviously, if $z \in P_{q}^{0}\left(\mathbb{Z}_{+}, X\right)$ and $\mu$ is a real number, then $\left(e^{i \mu n} z(n)\right)_{n \in \mathbb{Z}_{+}}$belongs to $A P_{0}\left(\mathbb{Z}_{+}, X\right)$. Now, we can apply Corollary 4 to finish the proof.

The following example is a concrete application of Theorem 5.

Example 6. Let $X$ be a complex Banach space, and let $A$ be a bounded linear operator acting on $X$. Consider the following two discrete Cauchy problems:

$$
\begin{gathered}
x_{j+1}=A x_{j}, \quad j \in \mathbb{Z}_{+}, \\
x_{0}=b, \\
y_{j+1}=A y_{j}+f_{j+1}, \quad j \in \mathbb{Z}_{+}, \\
y_{0}=0 .
\end{gathered}
$$

The solutions of (20) and (21) are (resp.) given by $x_{j}=T(j) b$ and $y_{j}=\sum_{k=0}^{j} T(j-k) x(k)$. Here $T(k):=A^{k}$. alent.

From Theorem 5 , the following two statements are equiv-

(1) For each $b \in X$ the solution of (20) decays exponentially, or, equivalently, there exist two positive constants $K$ and $v$ such that

$$
\|T(j) x\| \leq K e^{-v j}\|x\| \quad \forall x \in X .
$$

(2) For each $f \in A P_{0}\left(\mathbb{Z}_{+}, X\right)$ the solution of (21) belongs to $A P_{0}\left(\mathbb{Z}_{+}, X\right)$.

In fact, we can state a more general result concerning $q$ periodic discrete evolution families. To establish this result, we recall that a family $\mathcal{U}=\left\{U(n, m): n \geq m \in \mathbb{Z}_{+}\right\} \subset \mathscr{B}(X)$ is said to be $q$-periodic discrete evolution family if it satisfies the following properties.

(i) $U(n, n)=I$ and $U(n, m) U(m, r)=U(n, r)$, for all $n, m, r \in \mathbb{Z}_{+}$with $n \geq m \geq r \in \mathbb{Z}_{+}$, where $I$ is the identity operator on $X$.

(ii) $U(n+q, m+q)=U(n, m)$, for all $n \geq m \in \mathbb{Z}_{+}$. 
It is said to be uniformly exponentially stable if there exist the positive constants $K$ and $\nu$ such that

$$
\|U(n, m)\| \leq K e^{-\nu(n-m)} \quad \forall m \geq n \in \mathbb{Z}_{+} .
$$

Also, the family $\mathscr{U}$ is said to be $A P_{0}\left(\mathbb{Z}_{+}, X\right)$ exact admissible, if for every $z \in A P_{0}\left(\mathbb{Z}_{+}, X\right)$ the sequence $\left(\sum_{k=0}^{n} U(n\right.$, $k) z(k))_{n \in \mathbb{Z}_{+}}$belongs to $A P_{0}\left(\mathbb{Z}_{+}, X\right)$.

The discrete evolution semigroup $\mathscr{T}=\left\{\mathscr{T}(n), n \in \mathbb{Z}_{+}\right\}$ associated with the evolution family $\mathcal{U}$ on $A P_{0}\left(\mathbb{Z}_{+}, X\right)$ is defined by

$$
(\mathscr{T}(n) z)(r)= \begin{cases}U(r, r-n) z(r-n), & \forall r \geq n, \\ 0, & \text { otherwise. }\end{cases}
$$

As in Lemma 1 it can be proved that it acts on $A P_{0}\left(\mathbb{Z}_{+}, X\right)$.

Theorem 7. Let $\mathcal{U}=\left\{U(n, m): n \geq m \in \mathbb{Z}_{+}\right\}$be a q-periodic evolution family of bounded linear operators on $X$. The following statements are equivalent:

(1) $\mathcal{U}$ is uniformly exponentially stable.

(2) The evolution semigroup $\mathscr{T}$ associated with $\mathcal{U}$ is uniformly exponentially stable.

(3) $\mathcal{U}$ is $A P_{0}\left(\mathbb{Z}_{+}, X\right)$ exact admissible.

(4) $\sup _{n \in \mathbb{Z}_{+}}\left\|\sum_{k=0}^{n} U(n, k) h(k)\right\|<\infty$, for all $h \in A P_{0}\left(\mathbb{Z}_{+}\right.$, $X)$.

The proofs of $(1) \Rightarrow(2) \Rightarrow(3) \Rightarrow(4)$ are similar to those in the semigroup case. For the proof of $(4) \Rightarrow(1)$ we use the following result from [13].

If for every $\mu \in \mathbb{R}$ and every $z \in P_{q}^{0}\left(\mathbb{Z}_{+}, X\right)$, one has

$$
\sup _{n \in \mathbb{Z}_{+}}\left\|\sum_{k=0}^{n} e^{i \mu k} U(n, k) z(k)\right\|:=M(\mu, z)<\infty,
$$

then the family $\mathcal{U}$ is uniformly exponentially stable.

\section{Acknowledgment}

The authors would like to thank the anonymous referees for their comments and suggestions on preliminary versions of this paper, which have led to a substantial improvement in its readability. In particular, we have completed the last section of this note at the suggestions of referees.

\section{References}

[1] J. van Neerven, The Asymptotic Behaviour of Semigroups of Linear Operators, vol. 88 of Operator Theory: Advances and Applications, Birkhäuser, Basel, Switzerland, 1996.

[2] C. Chicone and Y. Latushkin, Evolution Semigroups in Dynamical Systems and Differential Equations, vol. 70 of Mathematical Surveys and Monographs, American Mathematical Society, Providence, RI, USA, 1999.

[3] S. Clark, Y. Latushkin, S. Montgomery-Smith, and T. Randolph, "Stability radius and internal versus external stability in Banach spaces: an evolution semigroup approach," SIAM Journal on Control and Optimization, vol. 38, no. 6, pp. 1757-1793, 2000.
[4] O. Saierli, "Spectral mapping theorem for an evolution semigroup on a space of vector-valued almost-periodic functions," Electronic Journal of Differential Equations, vol. 2012, no. 175, pp. $1-9,2012$.

[5] C. Buşe, D. Lassoued, T. L. Nguyen, and O. Saierli, "Exponential stability and uniform boundedness of solutions for nonautonomous periodic abstract Cauchy problems. An evolution semigroup approach," Integral Equations and Operator Theory, vol. 74, no. 3, pp. 345-362, 2012.

[6] C. Buşe, S. S. Dragomir, and V. Lupulescu, "Characterizations of stability for strongly continuous semigroups by boundedness of its convolutions with almost periodic functions," International Journal of Differential Equations and Applications, vol. 2, no. 1, pp. 103-109, 2001.

[7] A. Zada, G. Rahmat, G. Ali, and A. Tabassum, "Characterizations of stability for discrete semigroup of bounded linear operators," International Journal of Mathematics and Soft Computing, vol. 3, no. 3, 2013.

[8] C. Buşe and O. Jitianu, "A new theorem on exponential stability of periodic evolution families on Banach spaces," Electronic Journal of Differential Equations, vol. 2013, no. 14, pp. 1-10, 2003.

[9] C. Corduneanu, Almost Periodic Oscillations and Waves, Springer, New York, NY, USA, 2009.

[10] A. S. Besicovitch, Almost Periodic Functions, Dover Publications, New York, NY, USA, 1955.

[11] R. G. Douglas, Banach Algebra Techniques in Operator Theory, vol. 179 of Graduate Texts in Mathematics, Springer, New York, NY, USA, 2nd edition, 1998.

[12] C. Buşe, A. Khan, G. Rahmat, and A. Tabassum, "Uniform exponentialstability for nonautonomous system via discrete evolution semigroups," to appear in Bulletin Mathématique de la Société des Sciences Mathématiques de Roumanie.

[13] C. Buşe, P. Cerone, S. S. Dragomir, and A. Sofo, "Uniform stability of periodic discrete systems in Banach spaces," Journal of Difference Equations and Applications, vol. 11, no. 12, pp. 1081$1088,2005$. 


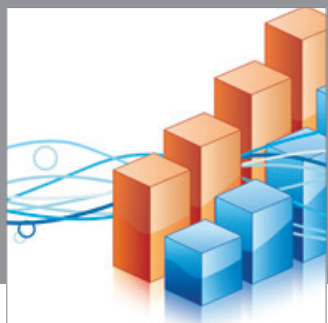

Advances in

Operations Research

mansans

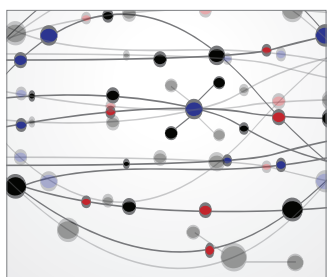

The Scientific World Journal
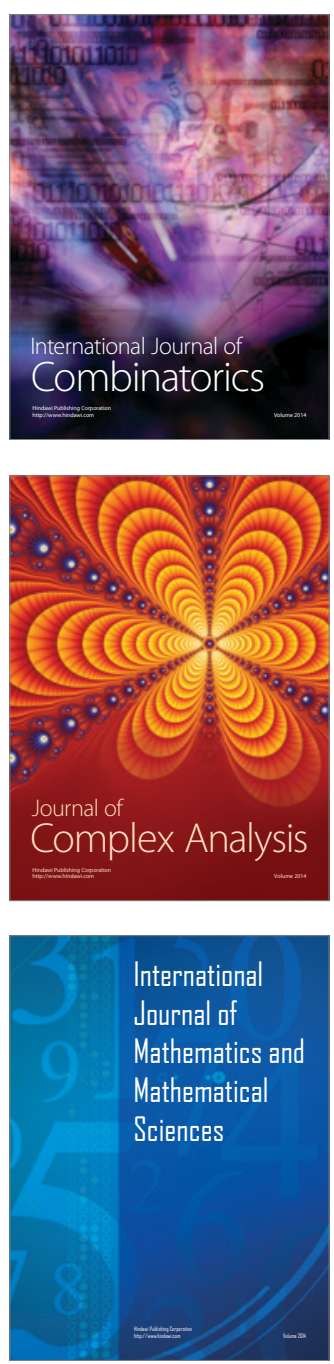
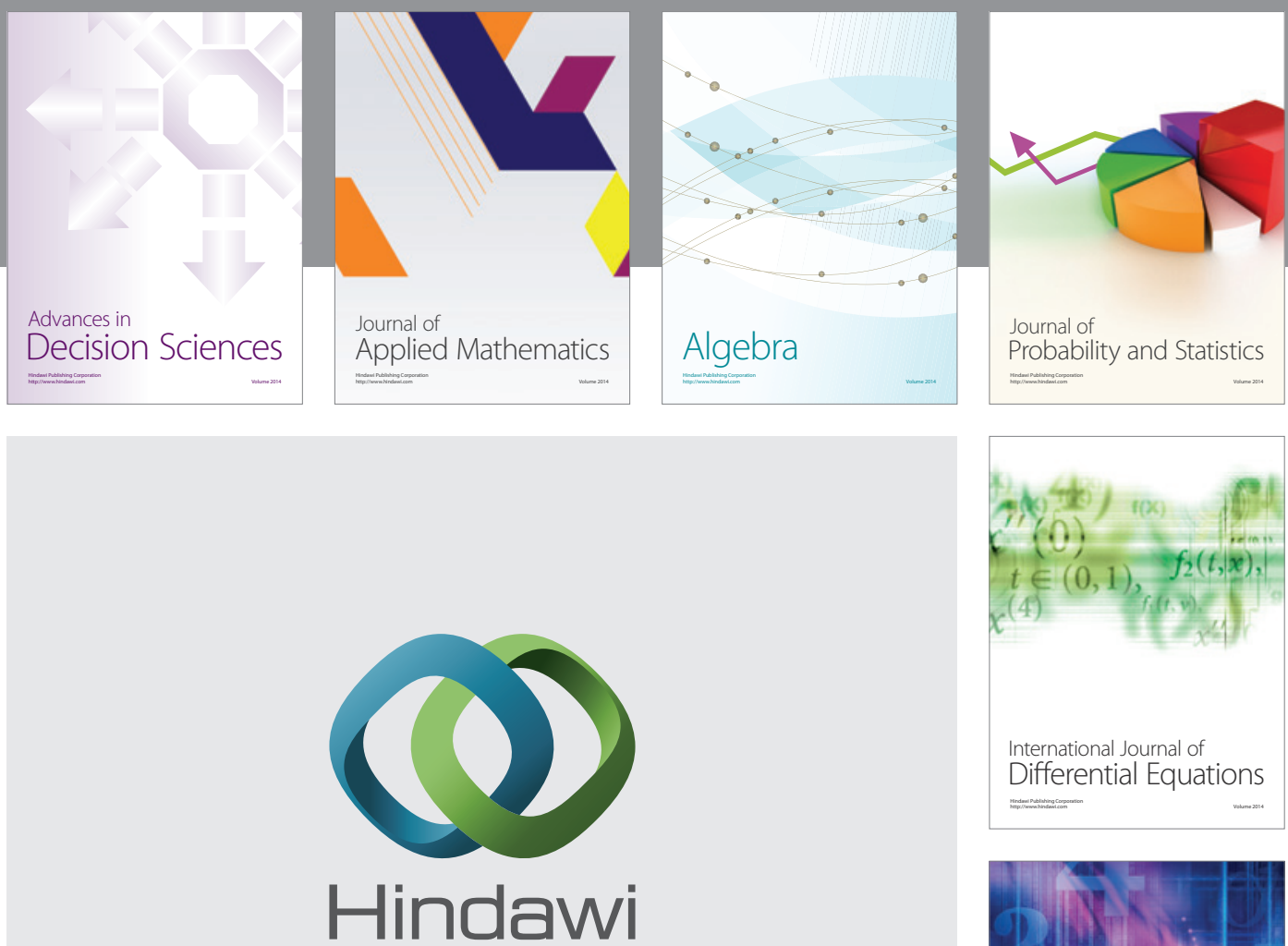

Submit your manuscripts at http://www.hindawi.com
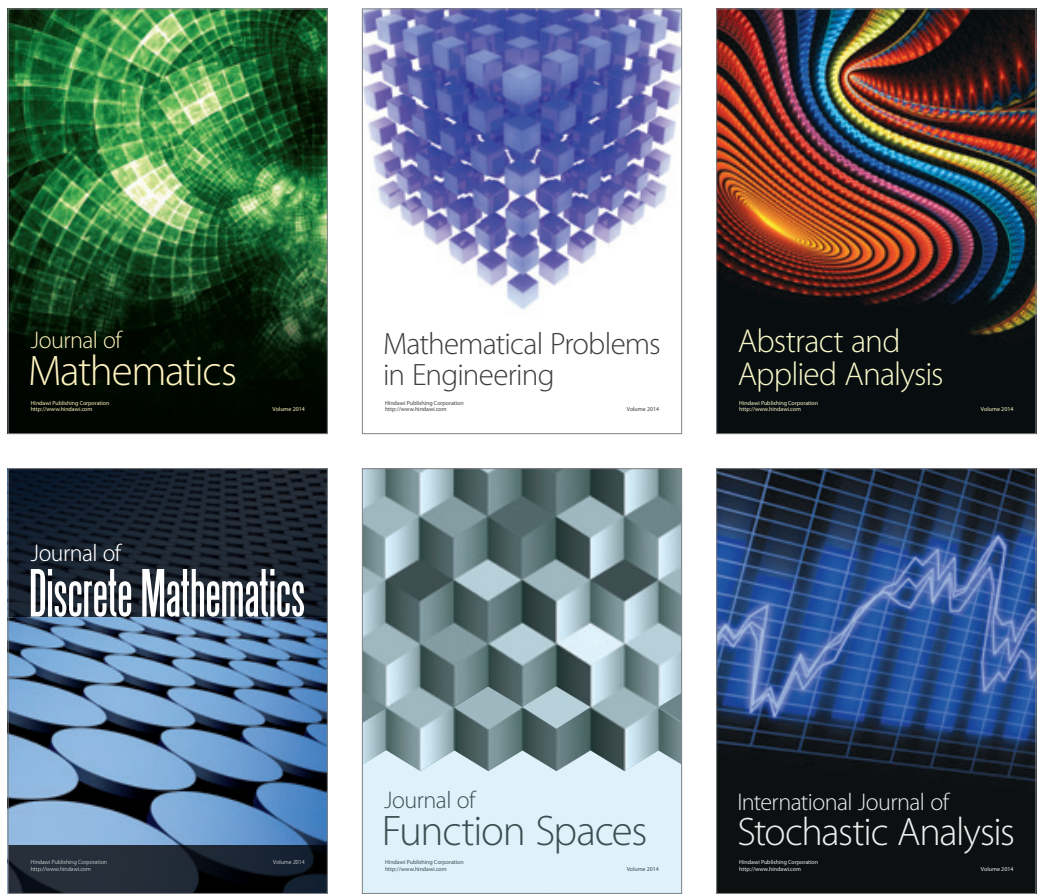

Journal of

Function Spaces

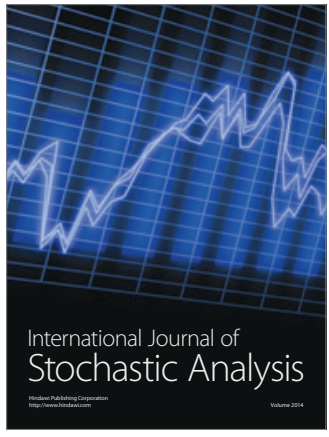

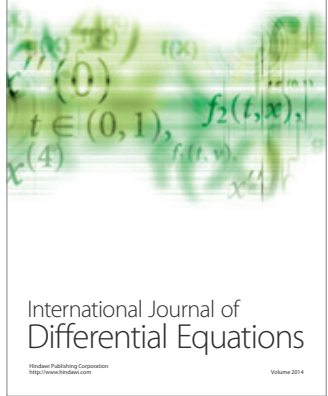
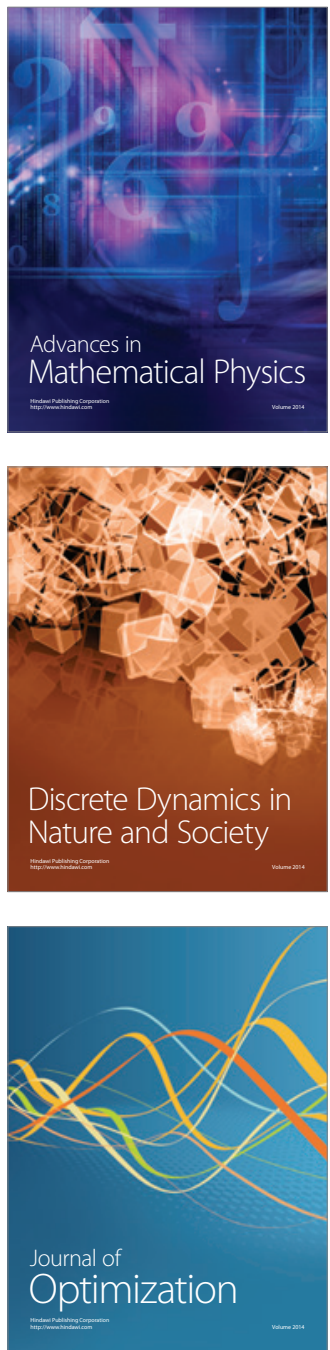